\title{
MURINE CORONAVIRUS TEMPERATURE SENSITIVE MUTANTS
}

\author{
Ralph S. Baric ${ }^{1 *}$, Mary C. Schaad ${ }^{1}$, Theodore Wei ${ }^{2}$ \\ Kaisong $\mathrm{Fu}^{1}$, Karen Ium ${ }^{1}$, Carol Shieh ${ }^{1}$ and \\ Stephen A. Stohlman 2
}

\author{
${ }^{1}$ Department of Parasitology and Laboratory Practice \\ School of Public Health \\ University of North Carolina at Chapel Hill \\ Chapel Hill, North Carolina 27599 \\ 2 Department of Neurology and Microbiology \\ University of Southern California \\ School of Medicine \\ Mckibben Annex 142 \\ 2025 Zonal Avenue \\ Los Angeles, California 90033
}

\section{INTRODUCTION}

Mouse hepatitis virus (MHV), a member of the Coronaviridae, contains a single-stranded, nonsegmented, plus-polarity RNA of $8.0 \times 10^{6}$ daltons molecular weight ${ }^{1}$. The $\sim 32 \mathrm{~KB}$ genomic RNA is organized into seven genetic regions each encoding one or more viral proteins 1,2 . In the virion, the RNA is enclosed in a helical nucleocapsid structure constructed from multiple copies of a 50-60KD phosphorylated nucleocapsid protein(N). The viral envelope is derived from modified host internal membranes and contains two virus-encoded glycoproteins designated $M$ (gp23) and $s(g p 180 / 90) 1,3$.

Upon entry into the host cell, the genomic RNA is translated into an RNA-dependent RNA polymerase which directs the synthesis of a full length negative-stranded RNA 4 . In turn, the negative-stranded RNA acts as template for the synthesis of seven virus specific mRNAs 4,5 . The most probable mechanism to explain the mechanism of MHV transcription involves the synthesis of a free leader RNA(s) which act in trans as a primer for mRNA synthesis 7,8,9,10,11. Unfortunately, little data is available concerning the location and function of individual viral genes which participate in RNA synthesis. It is suspected that one or more viral proteins are encoded which regulate negative-strand synthesis, leader RNA synthesis, mRNA synthesis and genome replication.

Temperature sensitive (ts) mutants of animal viruses are useful for assigning particular physiologic, biochemical and pathogenic functions to individual viral genes. Complementation analysis of MHV ts mutants suggest that at least six $\mathrm{RNA}^{-}$and two $\mathrm{RNA}^{+}$complementation groups are encoded in the MHV genome 12,13. The location and function of these complementation groups in viral transcription is not clear. In this article, we describe the isolation and characterization of several complementation groups of MHV-A59 which function in positive and/or 
negative-strand synthesis. Utilizing genetic recombination techniques, these data indicate that the four $\mathrm{RNA}^{-}$complementation groups used in this study map a linear array at the $5^{\prime}$ end of the genome in the $21 \mathrm{~KB}$ polymerase region, and also suggest that the RNA recombination frequency for the MHV-A59 genome may approach $25 \%$.

\section{METHODS}

\section{Virus and Cell Lines}

The A59 strain of mouse hepatitis virus (MHV-A59) was used throughout the course of this study. Virus was propagated and cloned three times in the continuous murine astrocytoma cell line (DBT). Prior to use in these experiments, cloned MHV-A59 at passage level six at $39^{\circ} \mathrm{C}$, was plaque purified $2 \mathrm{X}$ in $\mathrm{DBT}$ cells at $39^{\circ} \mathrm{C}$.

\section{Temperature Shift Experiments}

Cultures of DBT cells were infected at a MOI of 2 with different ts mutants and maintained at $32^{\circ} \mathrm{C}$. Following incubation at $32^{\circ} \mathrm{C}$ for 5.5 hrs, duplicate cultures were shifted to restrictive temperature by the addition of prewarmed media and virus progeny harvested at different times post-infection for analysis by plaque assay. In addition, intracellular RNA was extracted at 5.5, 7.5, 9.5, and 11.5 hrs postinfection and analyzed for the presence of viral mRNA with strand specific RNA probes representing the $N$ gene sequence ${ }^{13}$. Alternatively, filters were probed with strand specific RNA probes which specifically hybridize genome or (-)-stranded RNA.

\section{Recombination Test}

Various combinations of ts mutants were mixed and inoculated onto cells at a multiplicity of infection of 10 each. Plates were rocked every 15 mins for $1 \mathrm{hr}$ at room temperature and the inoculum removed. Individual wells were washed gently $2 \mathrm{x}$ with $2 \mathrm{mls}$ of warm PBS and incubated at $32^{\circ} \mathrm{C}$ for $16 \mathrm{hrs}$ in $2 \mathrm{mls}$ of dMEM containing $10 \%$ antibiotic/antimycotic. Virus progeny were harvested and frozen at $-70^{\circ} \mathrm{C}$ for future study. Each cross was titered at $32^{\circ} \mathrm{C}$ and $39.5^{\circ} \mathrm{C}$ by plaque assay and the recombination frequencies calculated as the percent of recombinant $\mathrm{ts}^{+}$virus present in the progeny utilizing techniques previously reported for picornaviruses 15,16 .

Recombination frequencies were standardized against a standard cross (LA7 $x$ LA9) that was included in each experiment to obviate day to day variation. LA7 and LA9 were chosen as controls because LA7 maps 7 to $8 \mathrm{~KB}$ from the $3^{\prime}$ end of the genome in the gp180/90 envelope glycoprotein gene while LA9 maps in the polymerase gene at the 5 'end of the genome $17,18,19,20$. The average recombination frequency for each cross was calculated from 5 to 10 individual crosses which had been standardized to the control cross. The ts mutants were arranged according to standard genetic practices.

\section{RESULTS}

\section{Isolation and Characterization of MHV-A59 Ts Mutants}

Mutants were screened for the ability to form plaques at the permissive, but not restrictive temperature, and only those isolates which showed a differential titer of at least $1 \times 10^{-3}$ were retained for further study. Fourteen ts mutants were isolated from MHV-A59 infected cells that had been treated with $20 \mathrm{ug} / \mathrm{ml}$ 5-azacytidine and an additional four mutants were isolated from 5-fluorouracil treated cultures. Two mutants were derived from cultures treated with either $350 \mathrm{ug} / \mathrm{ml}$ of 5fluorouracil (LA14, LA15) or with $450 \mathrm{ug} / \mathrm{ml}$ of the drug (LA16, LA18). The 
RNA phenotype of each mutant was analyzed at the nonpermissive

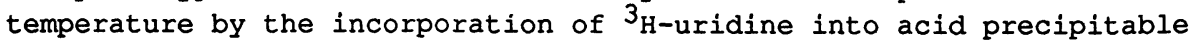
material from actinomycin D-treated virus-infected cells. Four of the ts mutants (LA7, LA12, LA13, NC5) were considered to be of RNA positive phenotype since levels of transcription at $39^{\circ} \mathrm{C}$ were at least $35 \%$ of the wildtype controls. The remaining 14 mutants were of the RNA phenotype since transcription at $32^{\circ} \mathrm{C}$ was less than $7 \%$ of the parental virus (Data not shown).

To determine the number of genetic functions represented in our panel of ts mutants, complementation analyses were performed. The complementation index for the ts mutants used in this study are summarized in Table 1. These data indicate that four $\mathrm{RNA}^{-}$( $\mathrm{A}, \mathrm{B}, \mathrm{C}$, and $D$ ) and one $\mathrm{RNA}^{+}$(E) complementation groups are represented within this panel of ts mutants. These data are compatible with previous results obtained by other groups with MHV-A59, JHM, or MHV-3 ts mutants. All RNA mutants tested were incapable of transcribing mRNA, (-)-stranded RNA or genome at the restrictive temperature (Date not shown).

TABLE 1. COMPLEMENTATION GROUPS OF MHV-A59

$\begin{array}{lll}\text { GROUP A } & - & \text { IHA3, IA6, IHA16, } \\ \text { GROUP B } & - & \text { IAA, IA8, IAI4, } \\ \text { GROUP C } & - & \text { IAA10 } \\ \text { GROUP D } & - & \text { IA18, NC4 } \\ \text { GROUP E } & - & \text { IA7, IAI2, NC5 }\end{array}$

\section{Virus Growth Curves Following Temperature Shift}

Temperature shift experiments immediately after the onset of positive strand RNA synthesis have elucidated the basic genetics of alphavirus transcription and replication. Duplicate cultures of cells were infected with different $\mathrm{RNA}^{-}$or $\mathrm{RNA}^{+}$ts mutants and incubated at $32^{\circ} \mathrm{C}$ for $5.5 \mathrm{hrs}$. One-half the cultures were shifted to restrictive temperature by the addition of prewarmed media, and virus progeny assayed at $7.5,9.5,11.5$, and 16.0 hrs post-infection. Shift to restrictive temperature blocked the release of infectious virus from complementation groups $A, C$ and D (RNA- mutants). In contrast, the replication of the $\mathrm{RNA}^{-}$group $\mathrm{B}$ mutant LA9 was unaffected by shift to restrictive temperature and continued to released infectious virus. The $\mathrm{RNA}^{+}$group $\mathrm{E}$ mutant was also blocked in the ability to release infectious virus after shift to restrictive temperature (Data not shown).

\section{Analysis of MHV RNA Synthesis following Temperature Shift}

Previous studies demonstrated the presence of two classes of $\mathrm{RNA}^{-}$ mutants defective in either an early (Group B) or late function (Groups $A, C, D, E)$ in virus replication. We next examined the ability of these mutants to transcribe mRNA following shift to restrictive temperature. Duplicate culture of cells were infected with ts mutants and maintained at $32^{\circ} \mathrm{C}$ for 5-6 hrs. One-half of the cultures were shifted to restrictive temperature by the addition of pre-warmed media, and intracellular RNA isolated at different times post-infection. The RNA was bound to nitrocellulose filters and hybridized with an $\mathrm{N}$ gene strand specific RNA probe which detects all viral positive-sensed mRNAs. As expected, the RNA ${ }^{+}$complementation group synthesized viral RNA after shift to restrictive temperature. Consistent with the inability to shed infectious virus, $\mathrm{RNA}^{-}$complementation groups $\mathrm{A}, \mathrm{C}$ and $\mathrm{D}$ were defective 
in mRNA synthesis following shift to restrictive temperature. Conversely, the group $\mathrm{E} \mathrm{RNA}^{+}$and group $\mathrm{B} \mathrm{RNA}^{-}$complementation groups continued to transcribe RNA at restrictive temperature. Complementation groups $A, C$ and $D$, but not $B$ or $E$, were also defective in the synthesis of genomic RNA (Table 2).

In cultures radiolabeled with ${ }^{32} \mathrm{P}$-orthophosphate, increasing amounts of viral mRNA were synthezied in group B-infected cells that were shifted to restrictive temperature at progressively later times post-infection (data not shown). These data indicate that the group B mutants were defective in an early event in virus transcription which effects the overall rates of positive strand synthesis later in infection.

TABLE 2. FUNCTIONAL ANALYSIS OF THE MHV-A59 COMPLEMENTATION GROUPS. GENOME MRNA (-) RNA LEADER FUNCTION

\begin{tabular}{|l|c|c|c|c|c|}
\hline GROUP A & - & - & - & - & CORE \\
\hline GROUP B & + & + & - & + & $(-)$ RNA \\
\hline GROUR C & - & - & $?$ & + & MRNA \\
\hline GROUP D & - & - & + & $-?$ & LEADER? \\
\hline GROUP E & + & + & + & + & $?$ \\
\hline
\end{tabular}

Complementation groups $A, B$ and $D$ were also examined for the ability to transcribe (-)-stranded RNA and leader RNA after shift to restrictive temperature. Infected cultures were maintained at permissive temperature for 5.5 hrs post-infection, and shifted to restrictive temperature. Intracellular RNA was extracted at $5.5,7.5,9.5$ and 11.5 hrs postinfection and probed for the presence of $(-)$-stranded RNA with strand specific RNA probes. Complementation groups $A$ and $B$ were blocked in the ability to transcribe $(-)$-stranded RNA at restrictive temperature. Under identical conditions the group D mutants continued to transcribe (-) RNA following shift to restrictive temperature (Table 2). These data indicate that groups $A$ and $B$ are defective in $(-)$ RNA synthesis at restrictive temperature while group $D$ mutants were blocked at a later stage in the virus growth cycle. In cultures monitored for leader RNA synthesis following shift to restrictive temperature, complementation that groups $A$ and $D$ were probably defective in the synthesis of small leader RNAs while groups $B, C$ and $E$ were not (Table 2).

\section{Recombination Mapping}

Utilizing standard genetic recombination techniques, poliovirus and apthovirus ts mutants have been arranged into an additive, linear, genetic map with mutants at different locations differing in physiologic function 16,17,18. To obtain a genetic recombination map, ts mutants from each complementation group were crossed with LA7 and LA9 and standardized to the standard cross (LA7 $x$ LA9 - 4.45 \pm 0.845 ). In addition, mutants from each group were crossed between mutants from other groups to provide unequivocal positioning. All the distances between complementation groups were within statistical limits and permit the construction of a genetic map (Figure 1). From the 5'end of the genome, the order of the complementation groups was: A, B, C, D, and E. Crosses between ts mutants in the same complementation group had characteristically low recombination frequencies. Not surprisingly, 
higher recombination frequencies were observed between mutants representing different complementation groups and were as high as $8.6 \%$ between group E (LA7) and group A (LA6) mutants. Group B and C mutants also had high recombination frequencies and support previous findings that these mutants map in the polymerase gene at the $5^{\prime}$ end of the genome $18,19,20$. It seems likely that the group $D$ mutants also map at the $3^{\prime}$ end of the polymerase gene since our data clearly indicate that this group is incapable of transcribing plus or minus-stranded RNA when maintained at restrictive temperature.

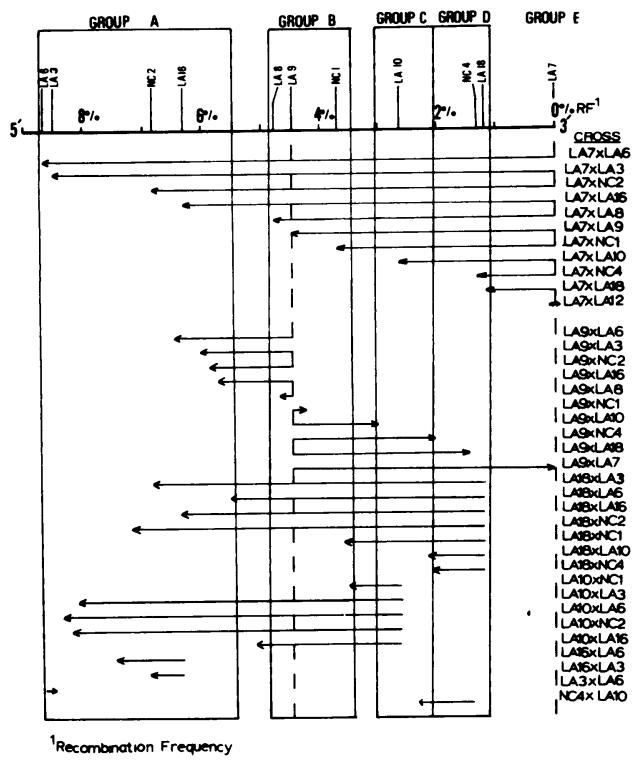

FIGURE 1. ESTABLISHING A GENETIC RECOMBINATION MAP OF THE MHV-A59 COMPLEMENTATION GROUPS

\section{DISCUSSION}

Little information is currently available concerning the number, location and function of the viral genes which participate in MHV transcription. Previous studies indicate that continous protein synthesis is a prerequisite for both positive and negative transcription and that MHV negative-strand synthesis is regulated temporally during infection 23 . Brayton et al 4,5 have described polymerase activities in MHV-infected cells which preferentially transcribe negative-stranded RNA, mRNA and genome. Temporal regulation of genome replication has been observed in $\mathrm{BCV}$-infected cells 28 . Other groups have also demonstrated in vitro mRNA and/or genome transcription and shown that anti-N mABs block-in vitro transcription 24 . These data are supported by studies in our laboratory suggesting that $\mathrm{N}$ is tightly associated with the transcription complex and leader RNA. These data suggest that distinct functions are encoded in the MHV genome which regulate negative-strand synthesis, leader RNA synthesis, mRNA synthesis and genome replication.

The $\mathrm{RNA}^{-}$mutants used in this study are incapable of transcribing any detectable levels of viral RNA at the restrictive temperature. However, temperature shift experiments after the onset of mRNA synthesis clearly revealed the presence of several classes of $\mathrm{RNA}^{-}$mutants (Table 2). Preliminary data suggest that group A mutants are defective in leader RNA, mRNA, genome and negative-strand transcription at restrictive temperature and probably represent a core function required during each stage in RNA synthesis. One possibility is that the group A mutants 
represent the protease encoded 5-6KB from the 5'end of the genome. Group C mutants synthesize leader RNA, but not mRNA at restrictive temperature, and probably encode a gene which functions during mRNA synthesis. Group $D$ mutants transcribe negative-sensed RNA, but not leader RNA, mRNA or genome at restrictive temperature, and probably a function during leader RNA transcription. Complementation group B clearly encodes a genetic function which effects the rate of positive strand RNA synthesis following shift to restrictive temperature. Our data suggests that the group $B$ allele functions during negative-strand synthesis. At least two additional $\mathrm{RNA}^{-}$complementation groups have been demonstrated by other investigators in the field. The function of these groups is currently unclear.

T 1 fingerprint analysis of recombinant virus suggest that complementation groups $A, B$ and $C$ map in the polymerase gene at the $5^{\prime}$ end of the genome and that the group $E$ mutants map in the $S$ envelope glycoprotein gene roughly $7-8 \mathrm{~KB}$ from the $3^{\prime}$ end of the genome $17,18,19,20$. Intratypic recombination frequencies between group $A$ and $E$ mutants approaches $8.6 \%$ or $17.2 \%$ assuming reciprocal crosses (Figure 2). Since LA7 maps $\sim 23-24 \mathrm{~KB}$ from the 5 ' end of the $32 \mathrm{~KB}$ MHV genome and group $A$ mutants probably do not map in the p28 protein encoded within the first $1.1 \mathrm{~KB}$ at the $5^{\prime}$ end of the genome, a $17.2 \%$ recombination frequency occurs over a $\sim 22.5 \mathrm{~KB}$ nucleotide domain or a $1 \%$ recombination frequency/1300 nucleotide pairs of RNA. Assuming an equivalent recombination frequency for the entire $32 \mathrm{kB}$ genome, these data predict that the recombination frequency for the MHV genome may approach 25\%. Genetic recombination maps for poliovirus and apthoviruses predict a recombination frequency of $2.2 \%$ for the entire viral genome $15,16,17,29$. Thus, MHV high frequency recombination approaches the reassortment frequency of segmented RNA viruses 37 and suggests that segmented RNA intermediates may reassort during mixed MHV infection. We have previously demonstrated the presence of small RNA intermediates bound to or dissociated from the replicative intermediate RNA ${ }^{9}$. It remains to be determined if the small RNAs represent the functional intermediates of $\mathrm{RNA}$ transcription and recombination.

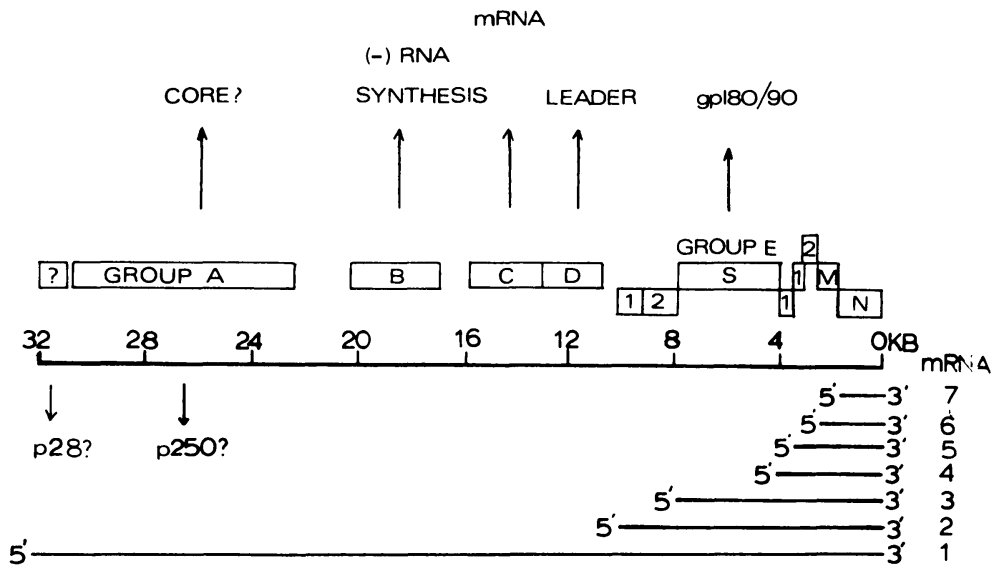

\section{FIGURE 2. TENTATIVE MAP DOMAINS OF EACH MHV-A59 COMPLEMENTATION GROUP}

Utilizing genetic recombination mapping techniques, we have formulated the first genetic map of the MHV-A59 ts mutants. The orientation of these complementation groups from the $5^{\prime}$ end of the genome was $A, B, C, D$ and $E$. Assuming a $1 \%$ recombination frequency/1300 nucleotide pairs of RNA, the nucleotide domains of each MHV-A59 complementation group can be calculated from the map domains illustrated in figure 1 (figure 2). Complementation groups $A, B, C$ and D 
definitively map in the polmerase region at the $5^{\prime}$ end of the genome. These data suggest that at least twelve genetic functions are encoded in the MHV genome including four in the polymerase region, two each in mRNAs2 and 5, and a single genetic function in mRNAs 3, 4, 6 and 7 (figure 2) $1,30,31,32,33$. The location of the two additional $\mathrm{RNA}^{-}$ complementation groups detected in other panels of ts mutants is unclear but could reside in the polymerase region or internally in the viral genome. By size analysis, the group A mutants map over a 7-8 KB stretch of RNA that could encode a protein of $\sim 250-300 \mathrm{kD}$ molecular weight. In vitro, the MHV genome is translated into a 250-300 KD polyprotein which is subsequently cleaved into a p28 and p220 protein 34,35. The p28 protein is encoded within the first $1.1 \mathrm{kB}$ from the $5^{\prime}$ end of the genome 35,36 . These data suggest that the group A mutants do not map in the p28 protein encoded at the $5^{\prime}$ end of the genome and suggest that a fifth genetic function is encoded in the polymerase region. We are currently determining the genetic location and function of additional ts mutants of MHV-A59.

\section{ACKNOWLEDGEMENTS}

The authors would like to express their appreciation to Phyllis Driscoll and Gillian Harris for excellent technical and secretarial assistance. This work was supported in part by a grant-in-aid from the American Heart Association (AHA 871135) and a grant from the National Institute of Health (AI 23946). This work was done during the tenure of an Established Investigatorship of the American Heart Association (AHA 890193) (RSB).

\section{REFERENCES}

1. Spaan, พ.J. et al (1988) J. Gen. Virol. 69:2939-2952

2. Siddell, S. (1983) J. Gen. Virol. 64:113-125

3. Sturman, I.S. et al (1980) J. Virol. 33:449-462

4. Brayton, P.R. et al (1982) J. Virol. 42:847-853

5. Brayton, P.R. et al (1984) Virol. 133:197-201

6. Lai, M.M.MC et al (1984) PNAS USA $81: 3626-3630$

7. Baric, R.S. et al (1983) J. Virol. 48:633-640

8. Makino, S. et al (1986a) PNAS USA 83:4204-4208

9. Baric, R.S. et al (1987) Virol. 156:342-354

10. Shieh, C-K., et al. (1987) Virology 156:321-330

11. Spaan, W.J. et al (1983) EMBO 2:1839-1844

12. Leibowitz, $J$. et al (1982) J. Virol. 42:1080-1087

13. Koolen, M.J. et al (1983) Viro. 125:393-402

14. Martin, J.P. et al (1988) J. Gen. Virol.

15. Mackenzie, J.S. et al (1975) J. Gen. Virol. 27:61-70

16. Lake, J.R. et al (1975) J. Gen. Virol. 27:355-367

17. Cooper, P.D. (1968) Virol. 35:584-596

18. Lai, M.M.C. et al (1985) J. Virol. 56:449-456

19. Keck, J.G. et al (1988b) J. Virol. $62: 1810-1813$

20. Keck, J.G. et al (1987) Virol. 156:331-341

21. Sawicki, D.I. and Sawicki, S.G. (1987) J. Virol. 25:19-27

22. Sawicki, S.G. and Sawicki, D.I. (1981) J. Virol. 115:161-172

23. Sawicki, S.G. and Sawicki, D.I. (1986) J. Virol. 57:328-334

24. Compton, S.R. et al (1987) J. Virol. 61: 1814-1820

25. Baric, R.S. et al (1988) J. Virol. 62: 4280-4287

26. Stohlman, S.A. et al (1988) J. Virol. 62:4288-4295

27. Baric, R.S. et al (1985)) Vir. Res. 3:19-33

28. Keck, J.G. et al (1988a) Vir. Res 9:343-356

29. Rirkkegaard, $K$. and Baltimore, D. (1986) Cell 47:433-443

30. Iuytjes, $\%$. et al (1988) Virol. In Press

31. Skinner, M.S. et al (1985) J. Gen. Virol 66:581-592 
32. Skinner, M.A. et al (1985) J. Gen. Virol 66: 593-596

33. Amstrong, $J$. et al (1984) Nature (London) 308:731-752

34. Dennison, M.R. and S. Perlman (1987) Virol. 60:12-18

35. Dennison, M.R. and S. Perlman (1988) Virol. 157:565-568

36. Soe, I. et al (1987) J. Virol. 61:3968-3976

37. Fields, B.N. (1981) Corr. Top. Micro. Imm. 91:1-24 